\title{
Structural analysis and molecular modeling of two anti- trichosanthin IgE clones from phage antibody library
}

\author{
LI Zong Dong, Yu Ren YUAN, Ming YEH ${ }^{1}$ \\ Shanghai Institute of Cell Biology, Chinese Academy of \\ Sciences, Shanghai 200031, China
}

\begin{abstract}
Recently we constructed a murine IgE phage surface display library and screened out two IgE (Fab) clones with specific binding activity to Trichosanthin (TCS). In this work, the $\mathrm{V} \varepsilon$ and $\mathrm{V} \kappa$ genes of the two clones were sequenced and their putative germline gene usages were studied. On the basis of the known 3D structure of Trichosanthin and antibody, molecular modeling was carried out to study the antigen-antibody interaction. The possible antigenic determinant sites on the surface of TCS recognized by both the clones Were analyzed, and the reaction forces between TCS and two Fab fragments were also analyzed respectively.
\end{abstract}

Key words: Anti-Trichosanthin IgE, molecular modeling, phage surface display library, antigenic determinants.

\section{INTRODUCTION}

In previous paper we had constructed a murine IgE phage surface display library for screening out high affinity anti-TCS IgE clones in the study of the antigenic determinants recognized by IgE. Two TCS- specific clones have been screened out from the library. One of them bound TCS with high specificity and the other was lower. In this paper we sequenced their $\mathrm{V} \varepsilon$ and $\mathrm{V} \kappa$ genes and their putative germline gene usages were also analyzed. Since the ordinates of TCS and antibody are known[l, 2], molecular modeling was used to study the TCS-IgE (Fab) interaction and the

1. Corresponding auther, e-mail: imceng@server.shcnc.ac.cn 


\section{Molecular modeling of murine anti-TCS phage Fab}

antigenic determinants that anti-TCS IgEs recognize. Because only a few crystal complexes composed of antigen- antibody have been successfully performed, molecular modeling is particularly useful in studying Ag-Ab interaction when crystal complex is not available. The antigenic determinants on TCS were predicted and the interaction between TCS and the two Fab clones were analyzed. These results were also compared with those obtained by us from anti-TCS IgE hybridomas[3, 4].

\section{MATERIAL AND METHODS}

\section{Plasmids}

Two Fob clones, Fab 2 and Fab 7, which contained $\mathrm{V} \varepsilon$ and $\mathrm{V} \kappa$, were established in our laboratory[5]. Both clones showed specific binding to TCS, and Fab 7 had higher binding activity.

\section{Sequence determination}

Plasmid DNAs were extracted by alkaline method, and the sequences were determined by the dideoxy (Sanger) sequencing method[6], using a TaqTrack Sequencing Kit (Promega). For each determination two to three independent gene clones were analyzed until consistent results were obtained. The sequences were analyzed with the DNA sequence database (National Center for Biotechnology Information, NIH). The complementary determing region (CDR) and framework region (FR) in deduced amino acid sequences were defined according to Kabat et al[7].

\section{Establishment of the $3 D$ model of the two Fab clones}

First, from the PDB data base one antibody sequence, 4FAB, which has the highest homology with our Fab clones, was chosen for the initial model. Second, all nonconserved amino acids within the $4 \mathrm{FAB}$ were changed to the corresponding residues in Fab 2 or Fab 7 by using software package TURBO. Third, the bad side-chain interactions were adjusted via molecular mechanics method. Finally, the revised model was subjected to further refinement of molecular dynamics and energy minimization by using X-plor software package. Thus the models of the two Fab clones were established for study.

The model building, energy minimization and the following study of interaction between TCS and Fabs were performed on a Silicon Graphics Iris Indigo2 workstation using the software packages TURBO and X-plor.

\section{The prediction of antigenic determinants on TCS}

The surface regions on TCS molecule complementary to that of the antigen-binding pocket formed by the CDRs of the two Fabs were examined. The side chain conformation was modified for reasonable interaction between residues of TCS and Fab in these regions, chiefly the hydrogen bonds and the van der Waals contacts (or especially those regions rich in hydrogen bonds). The surface regions of TCS that could well fit the antigen-binding pocket of Fab were taken as the possible antigenic determinant. Adjustment of the side chain conformation and the energy minimization of the whole structure were further performed.

\section{RESULTS}

\section{Sequence analysis}

The VH and VL gene sequences of Fab2 and Fab7 are listed as follows. Their 
VK OX1-1 - AA- -TTGTTCTCACCCAGTCTCCA-GCAATCAGTC FAB2VK -G-CA--AG-FAB2VH---:-

VH124-AGCCTGGGGCTTCAGTGCTGTCCTGCAAGGCTTCT

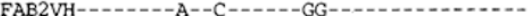

VH124-GGCTACACCTTCACCAGCTACTGGATGCACTGGGT

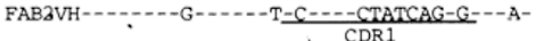

VH124-GAAGCAGAGGCCTGGACAAGGCCTTGAGTGGATCC FAB2VH---------AT-A----A-GA----A--------T-

VH124-GAGAGATTGATCCTTCTGATAGTTATACTAACTAC FAB2VH- - - ATTT-CA-C---ACA--GT-G------G---

VH124-AATCAAAAGTTCAAGGGCAAGGCCACATTGACTGT

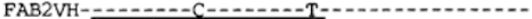

VH124-AGACAAATCCTCCAGCAC-AG-CCT-ACATGCAGC FAB2VH---GA---:---T-----G--T---G-A--C----

VH124-TCAGCAGCCTGACATCTGAGGACTCTGCGGTCTAT

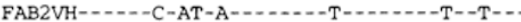

VH124 -TACTGTGCAAGA $>$

FAB2VH--.---G

DSP2.2- TATGATTACGAC
JH3
FAB2VH-AGGAAA-_TGGTTGCTTACTG
JH3 -GGGCCAAGGGACTCTGGTCACTGTCTCTGCA

JH3 -GGGCCAAGGGACTCTGGTCACTGTCTCTGCA
FAB2VH-

VKOX1-1 -TGCATCTCCAGGGGAGAAGGTCACCATGACCTGCA

FAB2VK -

VKOX1-1-GTGCCAGCTCAAGTGTAAGTTACATGCACTGGTAC FAB2VK -

VK OX1-1 -CAGCAGAAGC-AGGCACCTCCCCCAAAAGATGGAT FAB2VK -

VKOX1-1 -TTATGACACATCCAAACTGGCTTCTGGAGTCCCTG

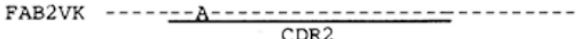

VKOX1-1 -CTCGCTTCAGTGGCAGTGGGTCTGGGACCTCTTAC FAB2VK -

VKOX1-1 -TCTCTCACAATCAGCAGCATGGAGGCTGAAGATGC FAB2VK - TCTCT-1

VKOX1-1 -TGCCACTTATTACTGCCAGCAGTGGAGTAGTA-AC FAB2VK - - - - G - .

VK $0 \times 1-1-\mathrm{CC}>$

FAB2VK

JK1 -TGGACGTTCGGTGGAGGCACCAAGCTGGAAATCAAA FAB2VK -GT-G-

Fig 1. Nucleotide sequences of the VH and VL of Fab2 and of their germline counterpart. Dashes signify identical nucleotides. CDR are according to Kabat et al. The boundaries of the CDR are indicated. FAB7

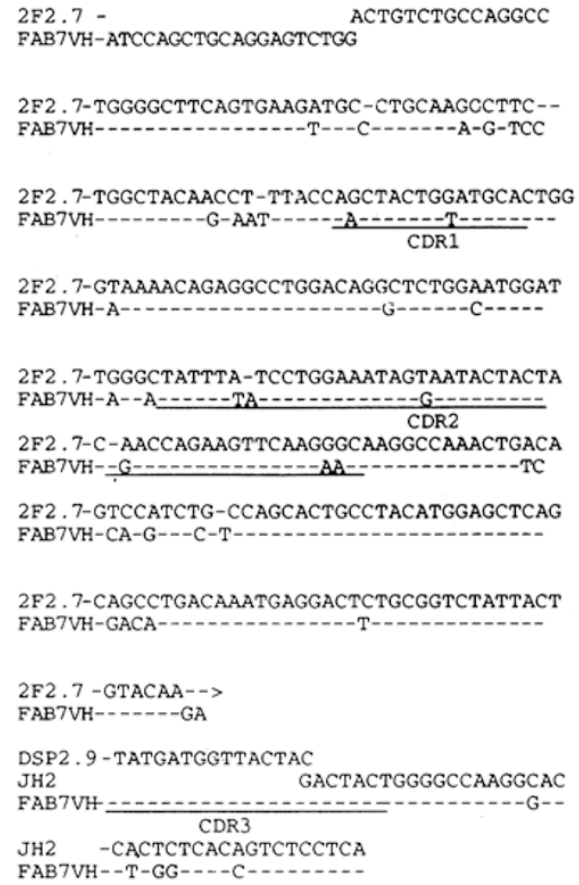

Fig 2. Nucleotide sequences of the VH and VL of Fab7 and of their germline counterparts.

Dashes signify identical nucleotides. CDR are according to Kabat et al. The boundaries of the CDR are indicated
VK ȮX1-1 -A-ATTGTTCTCACCCAGTCTCCAGCAATCA-GTC FAB7VK G-C---AG--

VKOX1-1 -TGCATCTCCAGGGGAGAAGGTCACCATGACCTGCA FAB7VK -

VK OX1-1 -GTGCCAGCTCAAGTGTAAGTTACATGCACTGG-TA FAB7VK $\frac{-10-1}{\text { CDR1 }}$

VKOX1-1-CC-AGC-AGAAG-CAGGCACCTCCCCCAAAAGATG

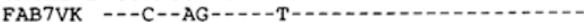

VK OX1-1 -GATTTATGACACATCCAAACTGGCTTCTGGAGTCC FAB7VK -

VKOX1-1-CTGCTCGCTTCAGTGGCAGTGGGTCTGGGA-CCTC FAB7VK -

VKOX1-1-TT--ACTCTCTCACAATCAGCAGCATGGAGGCTGA FAB7VK ---TA--

VKOX1-1 -AGATGCTGCCACTTATTACTGCCAGCAGTGGAG-FAB7VK -

VK OX1-1 -TAGTAA-CCC->

FAB7VK

JK1 -TGGACGTTCGGTGGAGGCACCAAGCTGGAAATCAAA

FAB7VK - 
comparison with putative germline genes are also given. For Fab2, the putative germline gene segments used by $\mathrm{V}$ region gene $(\mathrm{V}, \mathrm{D}, \mathrm{J})$ of heavy chain were $\mathrm{VH} 124$ of J558 family[8], DSP2.2, and JH3 respectively, and its light chain used OX1- 1 of VK4 family and JK1 (Fig 1).

For Fab7, the putative germline gene segments used by heavy chain gene for its V, D, J were 2F2.7 of J558 family, DSP2.9, and JH2 respectively. The Fab7 light chain used VK OX1-1 of VK4 family and JK1 (Fig 2).

\section{The molecular model of two Fabs and their complementarity to TCS}

Fig 3 and Fig 4 represent the molecular model of Fab2, Fab7 and their binding to TCS respectively. The two Fabs showed quite similar patterns in their interaction with TCS molecule. Two molecular surface regions in TCS were found to form extensive interactions with the CDRs of the two Fabs. They were thus to be predicted as the possible antigenic determinants, or allergenic determinants, on TCS. The first region is a discontinuous one, composed of Thr204 - Glu210 and Thr226Asn227, which are brought together by folding of the TCS polypetide chain to form an antigenic determinant. The second region is continuous, Lys173-Thr 178. The side chains of the residues in these two regions of TCS have formed a number of $\mathrm{H}$ bonds with the polar amino acid residues from L1, L3, H2, H3 of CDRs of the two Fabs. In addition, the CDR3 region of Fab2 is relative long and contained a plenty of aromatic amino acid.

The analysis of hydrogen bonding interactions between two Fabs and TCS

Tab 1 and Tab 2 give the H-bonds formed between Fabs and TCS region 1 and region 2 respectively. Results showed quite a number of hydrogen bonds were formed

Tab 1. The H-bond formation between Fab2 and region 1, 2 of TCS

\begin{tabular}{lccrc}
\hline Region 1 & Atom 1 in TCS & Atom 2 in Fab7 & (CDR) & distance $\left({ }^{\circ} A\right)$ \\
\hline & Thr204 OG1 & Asn50 OD1 & H2 & 2.91 \\
& Asn205 OD1 & Asn50 ND2 & H2 & 2.62 \\
& Asn206 OD1 & Arg98 NH1 & H3 & 3.00 \\
& Gln208 OE1 & Ser91 OG & L3 & 2.78 \\
& Gln210 OE2 & Asn59 ND2 & H2 & 3.06 \\
& Thr226 OG1 & Ser28 OG & L1 & 3.11 \\
& Asn227 OD1 & Ser92 OG & L3 & 3.14 \\
\hline & & & & \\
Region 2 & Atom 1 in TCS & Atom 2 in Fab7 & (CDR) & distance $\left({ }^{\circ}\right.$ A $)$ \\
\hline & Lys173 NE & Asn53 ND2 & H2 & 2.82 \\
& Arg174 NH2 & Lys57 NZ & H2 & 3.14 \\
& Asp176 OD2 & Arg98 NH1 & H3 & 3.06 \\
& Asp176 OD1 & Try31 OH & L1 & 2.66 \\
& Lys177 NE & Lys93 NZ & L3 & 3.16 \\
& Thr178 OG1 & Ser30 OG & L1 & 3.14 \\
\hline
\end{tabular}


Li ZD et al.
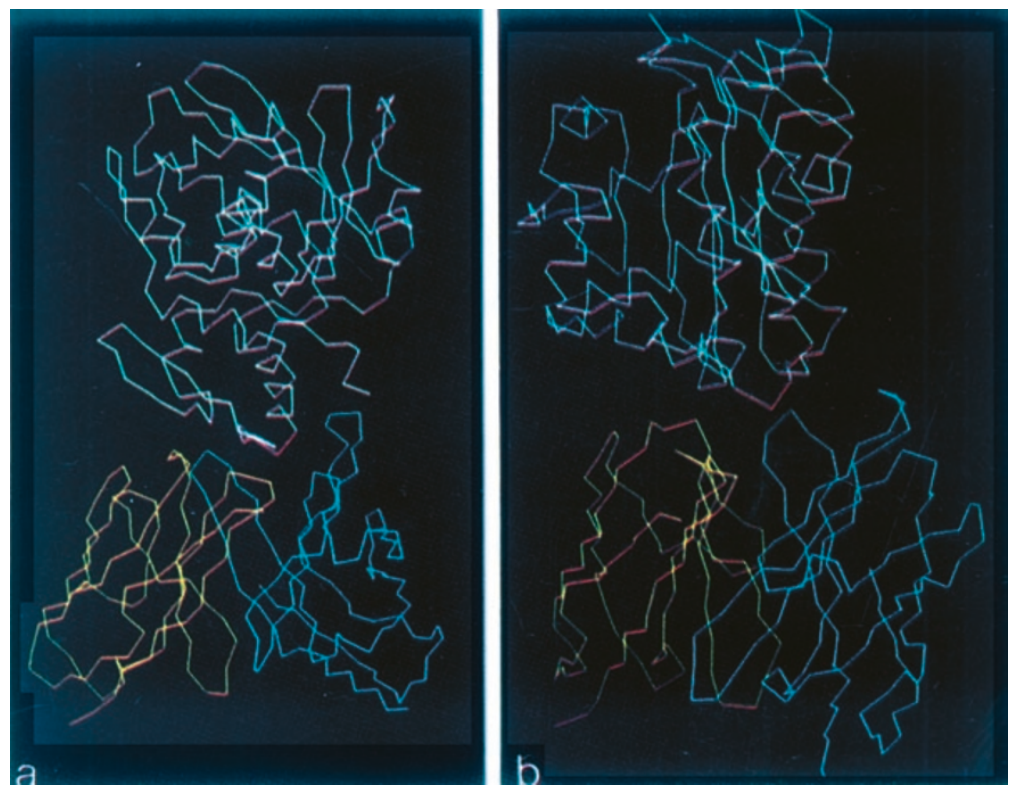

Fig 3. Molecular model showing the interaction between TCS and Fab2. The yellow chain and blue chain represent the $\mathrm{V}$ region of light chain and heavy chain of Fab 2 respectively. The white chain represents TCS. Rigion 1 and Rigion 2 are shown in red.

a. Fab2 binding to region 1 of TCS. b. Fab2 binding to region 2 of TCS.
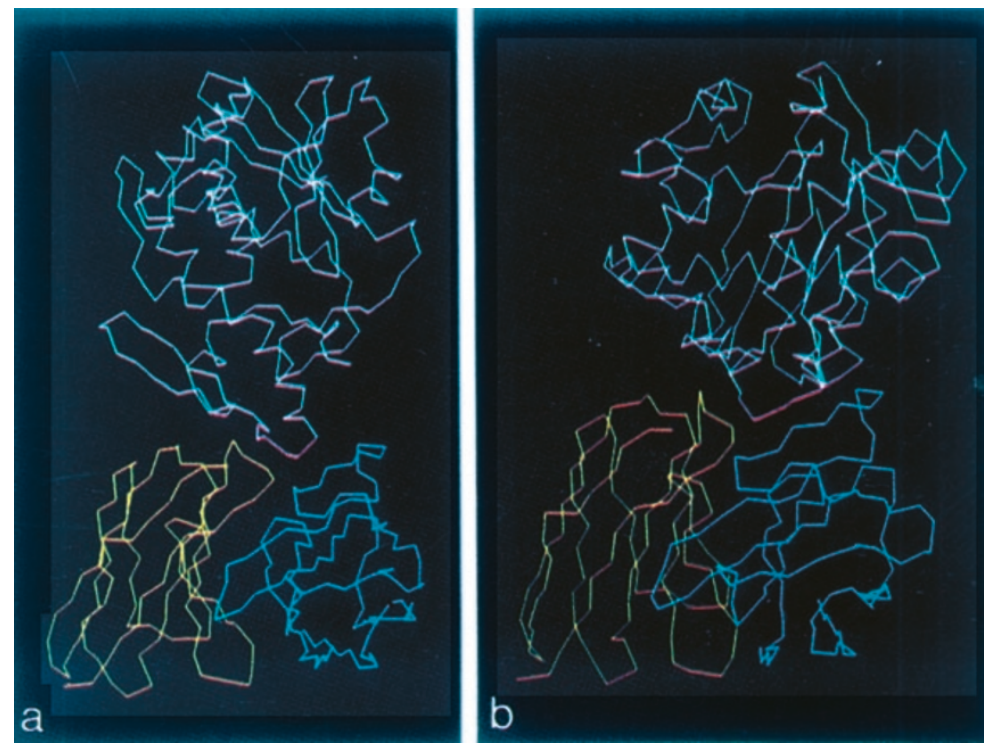

Fig 4. Molecular model showing the interaction between TCS and Fab 7. The yellow chain and blue chain represent the $\mathrm{V}$ region of light chain and the heavy chain of Fab 2 respectively. The white chain represents TCS. Region 1 and region 2 are shown in red. a. Fab7 binding to region 1 on TCS. b. Fab7 binding to region 2 on TCS. 
Molecular modeling of murine anti-TCS phage Fab

Tab 2. The H-bond formation between Fab 7 and region 1, 2 of TCS

\begin{tabular}{lcccc}
\hline Region 1 & Atom 1 in TCS & Atom 2 in Fab7 & $(\mathrm{CDR})$ & distance $\left({ }^{\circ} \mathrm{A}\right)$ \\
\cline { 2 - 5 } & Thr204 OG1 & Ser55 OD1 & H2 & 3.00 \\
& Asn205 OD1 & Try51 OH & H2 & 2.71 \\
& Asn206 OD1 & Try100 OH & H3 & 3.12 \\
& Gln208 OE1 & Gln90 OE1 & L3 & 3.02 \\
& Gln210 OE2 & Asn66 OD1 & H2 & 2.96 \\
& Thr226 OG1 & Ser28 OG & L1 & 3.02 \\
& Asn227 OD1 & Arg93 NH2 & L3 & 2.69 \\
\hline Region 2 & & & \\
& Atom 1 in TCS & Atom 2 in Fab7 & CDR & distance $\left.{ }^{\circ} \mathrm{A}\right)$ \\
\cline { 2 - 5 } & Lys173 NE & Asn54 ND2 & H2 & 2.98 \\
& Arg174 NH2 & Tyr97 OH & H3 & 2.80 \\
& Asp176 OD2 & Tyr101 OH & H3 & 2.94 \\
& Asp176 OD1 & Try31 OH & L1 & 2.88 \\
& Lys177 NE & Tyr103 OH & H3 & 2.88 \\
& Thr178 OG1 & Ser30 OG & L1 & 3.13 \\
\hline
\end{tabular}

between the two regions of TCS and Fabs, which supports the possible presence of antigenic determinants on region 1 and region 2 of TCS.

\section{DISCUSSION}

\section{Germ-line gene usages of the Fabs}

From the results here, we found that the two VH genes of heavy chains were all from J558 family but different germ-line gene segments, while the VL gene of light chain used the same germ-line gene segment OX1-1 of VH4 family and the same Jk gene segment JK1. The heavy chain usages showed variation, but light chain gene usags were more conserved. This result is quite similar to our previous results from hybridomas[3], although one was resulted from in vivo produced IgE rescued in hybridomas and one was from in vitro assembled Fabs. It supports our previous proposal that in anti-TCS IgEs, light chain may play more important role in binding to TCS than heavy chain. However, further investigations are needed.

\section{Considerations in searching for the antigenic determinant on TCS}

In searching for the antigenic determinant, several factors should be considered: 1) The determinant should protrude from the molecule surface. 2) There should be no obvious space block if it binds to the Fab fragment. 3) A number of non-bond reaction forces should be formed between Fab and antigen, such as hydrogen bonding or van der Waals force. Having examined the surface of the TCS molecule, we found that there were two sites that might be the antigenic determinants. After the Ag-Ab complex models were established, the analysis of $\mathrm{H}$-bonding indicated that quite a number of H-bonds would be formed between them. In comparison with region 2 as determinant, region 1 was of more possibility, since the space structure of region 1 is 
more complementary to that of antigen combining site formed by the CDRs of Fab and and less adjustments are required when establishing the Ag-Ab complex model. This result is also similar to the result we got from hybridoma before[4]. It seems that antibodies selected from phage antibody library may reflect the properties of antibodies selected by antigen in vivo at least to ceratin extent.

\section{In vivo antibody repertoire and in vitro antibody library}

From the comparison of the available antibody structures and their sequences, the "canonical structure model" has been proposed. Antibodies have only a few main-chain conformations for each hypervariable region, and most sequence variation would modifiy the surface provided by the side chains. Based on these surface changes resulted from sequence variation, different antigen combining sites are formed and hence different antigen recognition activity due to complementarity and mutual interaction forces. The relationship between structure and antigen recognition in Ag-Ab interaction is applicable to both in vivo and in vitro generated antibodies. During the in vivo generation of diversity, the pairing of different heavy chains with different light chain is also one of the important mechanisms. A specific antibody profile induced in an in vivo immune response is due to the selection by antigen of the specific antibody clones from the B lymphocyte repertoire. In phage antibody library, the $\mathrm{VH}$ in Fab is from the heavy chain $\mathrm{V}$ gene of a $\mathrm{B}$ cells and so does the VL. In a word, all the VH, VL genes in phage library come from vivo. The only difference is the light chain gene and heavy chain gene are assembled at random in vitro. They are not the original VH-VL pair in a B cell. However, since the antigen selection princple can also be applicable to in vitro, therefore we think that the specific antibodies screened out from a phage library could reflect the properties of in vivo antibody profile at least to a certain extent.

\section{Aromatic amino acids and polyreactivity}

In other report[9] involving analysis of the polyreactive IgEs, it showed that the CDR3 region of these antibodies contained more aromatic amino acid residues. Aromatic amino acids in CDR3 may play a significant role in polyreactivity of these antibodies. It can provide several forces for antibody binding, such as $\pi$ - $\pi$ stacking, $\mathrm{H}$-bond, and van der Waals force. Therefore, it was suggested that the presence of more aromatic amino acid in CDR3 region was responsible for the polyreactivity of these antibodies. In Fab 2, there are more aromatic amino acid residues in CDR3 in comparison with that in Fab 7. Fab 2 bound TCS with lower ELISA value in our previous report[5], while its nonspecific binding with other antigens (OVA, BSA) were higher. The richness of aromatic amino acid residues in CDR3 of Fab 2 may provide an explanation to its lower affinity to TCS. 
Molecular modeling of murine anti-TCS phage Fab

\section{A CKNO WLEGMENT}

The work was supported by the Director Grant of Shanghai Institute of Cell Biology and World Laboratory. We are most grateful to Professor Zong Xian XIA of Shanghai Institute of Organic Chemistry for her great help in using the computer workstation.

\section{REFERENCES}

[1] Xia ZX, Zhang L, Zhang ZM, Wu S, Dong YC. The three- dimensional structure of trichosanthin refined at 2.7 A resolution. Chinese J Chem 1993; 11:280-8.

[2] Zhang L, Xia ZX, Wu S, Dong YC. The further crystallographic refinement of trichosanthin at 2.7 A resolution and the comparison among the three molecular structures in two crystal forms. Chinese J Chem 1994; 12:223-30.

[3] Wang Y, Yeh M.Molecular Characteriation of the V regions of four IgE antibodies specific for Trichosanthin. Immunology 1996; 89:316-23.

[4] He YN, Xia ZX, Wang Y, Ji YY, Yeh M. Prediction of antigenic determinants of trichosanthin by molecular modelling. Cell Research 1996; 6:93-100.

[5] Li ZD, Yeh M. Construction and diversity analysis of a murine IgE phage surface display library. Cell Reseach 1997; 7:161-70.

[6] Sambrook J, Fritsch EF, Maniatis T. Molecular Cloning. Cold Spring Harbor Laboratory Press 1989.

[7] Kabat EA, Wu TT, Perry HM, Gottesman KS. Sequences of Protein of Immunological Interest. Public Health Service, NIH 1991.

[8] Cohen JB, Givol D. Allelic immunoglobuliln VH gene in two mouse strains: possible germline gene recombination. EMBO J 1983; 2:2013-8.

[9] Droupadi PR, Varga JM, Linthicum DS. Mechanism of allergenic cross-reactions-IV. Evidence for participation of aromatic residues in the ligand binding site of two multi-specific IgE monoclonal antibodies. Mol Immunol 1994; 31:537-48.

Received 27-8-1997. Revised 31-10-1997. Accepted 1-11-1997. 